\title{
ANÁLISE DE CONSERVAÇÃO DE ENERGIA ELÉTRICA NAS INDÚSTRIAS DO SETOR METAL-MECÂNICO DO ALTO VALE DO ITAJAÍ
}

\section{ANALYSIS OF ELECTRICAL ENERGY SAVING IN THE INDUSTRIES OF THE METAL-MECHANICAL SECTOR OF THE UPPER ITAJAÍ VALLEY}

\author{
Sidnei Luciano Raduenz \\ Rioar Automação Industrial, E-mail: sidnei@rioar.ind.br \\ Adriano Péres \\ Universidade Regional de Blumenau (FURB), E-mail: aperes@furb.br \\ Eduardo Deschamps \\ Universidade Regional de Blumenau (FURB), E-mail: edudes@furb.br
}

\begin{abstract}
Resumo: A disponibilidade de energia elétrica é hoje um dos principais fatores contribuintes para o desenvolvimento industrial de forma que a eficiência no uso deste recurso contribui para um desenvolvimento baseado na sustentabilidade. Este trabalho apresenta um estudo sobre as principais formas de obtenção de economia de energia em ambientes industriais tendo como referência as principais cargas do processo produtivo do setor metal-mecânico do Alto Vale do Itajaí, porém, sem considerar estudos de viabilidade financeira.
\end{abstract}

Palavras-chave: Aumento da eficiência energética, Substituição de equipamento menos eficiente, Economia de energia elétrica.

\begin{abstract}
The availability of electric energy today is a major factor contributing to industrial development so that the efficiency in the use of this resource contributes to a development based on sustainability. This paper presents a study of the main ways of achieving energy savings in industrial environments with reference to the main charge of the production process of the metal-mechanical sector of the Upper Itajaí Valley, but, without considering financial feasibility studies.
\end{abstract}

Key-words: Increasing electric efficiency, Substitution of less efficient electric equipment, Economy of electric energy. 


\section{INTRODUÇÃO}

A energia elétrica desempenha um papel fundamental na vida humana, servindo de alicerce para o atual modelo de desenvolvimento da sociedade, cujo desperdício vem se apresentando como um dos principais problemas a ser enfrentado. Uma sociedade, cujo desenvolvimento vise a sustentabilidade, deve enfocar o uso racional dos recursos naturais de forma a evitar o desperdício em função do mau uso da energia elétrica.

O uso da energia elétrica de forma eficiente apresenta-se como um novo paradigma a ser seguido. A mudança de hábitos e atitudes para a obtenção da eficiência energética em instalações industriais apresenta-se como um dos maiores obstáculos a serem vencidos.

O desenvolvimento da sociedade e a proteção ao meio ambiente são os dois maiores problemas globais que a humanidade está enfrentando, porém, ambos estão intimamente ligados de forma que o desenvolvimento segue como o principal meio de degradação do meio ambiente.

Com base nesta avaliação, a definição de meios ou metodologias de diagnóstico e análise da conservação de energia nas indústrias alcança o intuito de promover o uso da energia elétrica de modo a diminuir ao máximo as perdas e o desperdício buscando uma maior eficiência do sistema energético. Uma maior eficiência no sistema energético reflete o uso racional da energia, bem como, na minimização da necessidade de investimentos em novos sistemas de geração e conseqüentemente, redução dos impactos ambientais.

A busca pela eficiência energética nas indústrias do Alto Vale do Itajaí - mais especificamente no setor metal-mecânico - através da adoção de métodos de diagnóstico e análise da conservação de energia poderá contribuir para o crescimento econômico da região.

Obtêm-se em conseqüência da diminuição do consumo de energia elétrica a contribuição para a postergação de investimentos das empresas e da concessionária em novos sistemas de geração e distribuição além do aumento da competitividade.

\subsection{O ALTO VALE DO ITAJAI}

O desenvolvimento do Alto Vale do Itajaí está diretamente relacionado com a utilização de energia elétrica ou a sua transformação numa forma de energia mais apropriada para o processo produtivo. Assim sendo, a busca da eficiência energética e a redução de perdas torna-se de grande importância para o desenvolvimento industrial da região.

A região do Alto Vale do Itajaí possui 3 Pequenas Centrais Hidroelétricas ( $\mathrm{PCH}$ 's), sendo uma em Ibirama com capacidade de geração de $2 \mathrm{MW}$ e duas em Taió com capacidade de geração de 1,8MW e 0,6MW já interligadas ao sistema nacional de distribuição de energia. 
A capacidade instalada da CELESC, com 5 subestações no Alto Vale é de $128 \mathrm{MW}$, sendo a demanda média de $72 \mathrm{MW}$, ou seja, 56,3\% da oferta. A Figura 1 explana a situação do fornecimento de energia na região do Alto Vale do Itajaí.

O setor industrial metalmecânico apresenta-se como o maior consumidor de energia elétrica com um consumo mensal aproximado de $16,5 \mathrm{GWh}$ equivalente a $55,8 \%$ do consumo mensal da região.

Apenas as três maiores empresas do setor metal-mecânico do Alto Vale representam juntas, uma demanda mensal de 7,6MW de energia elétrica, ou seja, 10,6\% da demanda total do setor industrial ${ }^{1}$.

Cabe ressaltar que, conforme Ferreira (2003) não se sabe ao certo a fonte energética para a geração de energia em Santa Catarina, pois a CELESC é uma concessionária de energia que esta interligada ao setor elétrico brasileiro. $\mathrm{O}$ autor complementa que a CELESC produz apenas 3\% da energia consumida no Estado, sendo que os outros $97 \%$ são comprados de outras concessionárias.

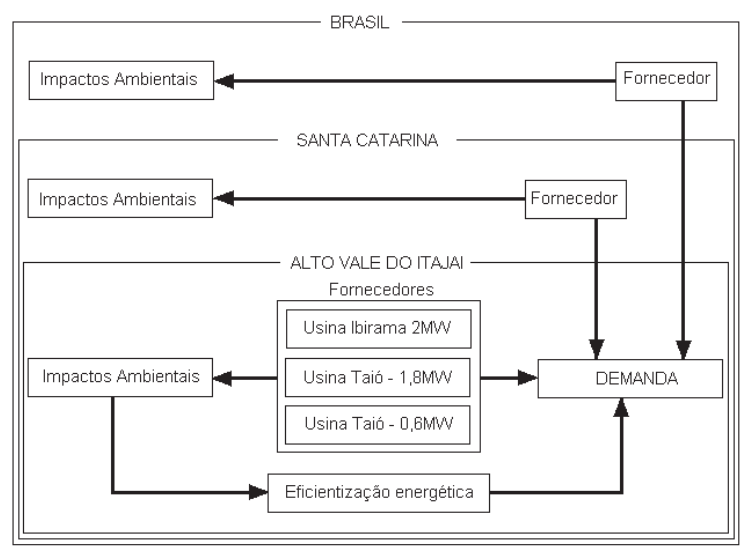

Figura 1: Fornecimento energia no Alto Vale do Itajaí

\section{METODOLOGIA DA PESQUISA}

Esta pesquisa utilizou, de acordo com Rauen (2002) o método de seleção de amostragem intencional, onde são escolhidos casos que representem o bom julgamento da população sob algum aspecto, através de estratégia adequada. Foram consideradas empresas componentes da amostragem a ser pesquisada as empresas do setor metal-mecânico do Alto Vale do Itajaí que apresentavam uma demanda contratada superior a 1 MW2.

${ }^{1}$ Dados obtidos com a concessionária de energia elétrica - CELESC em sua Agencia Regional de Rio do Sul 
A pesquisa bibliográfica tornou-se a base para o desenvolvimento da pesquisa de campo nas empresas selecionadas. A elaboração das perguntas do questionário e das entrevistas está fundamentada em recomendações e metodologias de diagnóstico energético do PROCEL - Programa Nacional de Conservação de Energia Elétrica.

Através de entrevistas com os responsáveis pelo gerenciamento de energia elétrica e visitas aos setores das empresas selecionadas foi possível, com as informações obtidas, verificar nas literaturas as principais formas de se obter o melhor rendimento dos equipamentos e instalações. Cabe ressaltar o registro das ações tomadas pelas empresas no intuito de promover a eficiência energética em seus parques fabris.

As perguntas efetuadas na pesquisa tiveram tratamento estatístico de forma a se obter conclusões diretas sobre a situação da conservação de energia elétrica no Alto Vale do Itajaí, porém tornou-se necessário o tratamento qualitativo das informações obtidas relacionadas com a investigação sobre os métodos empregados pelas empresas da região para aumentar a eficiência energética em suas plantas.

Em função do objetivo deste trabalho não foram consideradas na pesquisa de campo a análise da curva de demanda e a análise do sistema de tarifação das empresas selecionadas. A análise foi direcionada ao consumo de energia e as possibilidades de eficientização mantendo-se a necessidade de se efetuar uma análise da unidade industrial no que diz respeito à mesma permitir sua operação em faixas de eficiência adequadas, em seu processo produtivo.

\subsection{APRESENTAÇÃO DA EMPRESA SELECIONADA}

Para a realização da pesquisa, apenas três empresas da região apresentavam uma demanda superior a $1 \mathrm{MW}$, desta forma selecionou-se a empresa que apresentou a maior demanda contratada pelos motivos abaixo expostos:

a) apresentava os processos de usinagem e fundição empregados em larga escala no seu processo produtivo e que intrinsecamente apresentam cargas representadas por motores elétricos, sistemas de iluminação, sistemas pneumáticos, transformadores elétricos, sistemas de ventilação e fornos industriais; ressalta-se que nos escritórios ou áreas administrativas os sistemas de iluminação e ar condicionado são as cargas predominantes.

b) a empresa apresentava uma equipe técnica apta para auxiliar no levantamento de dados.

c) os dados obtidos poderiam ser extrapolados para as demais empresas com referência aos seus processos. 


\section{CONSERVAÇÃO DE ENERGIA EM AMBIENTES INDUSTRIAIS}

É preciso que a tecnologia e as ferramentas adequadas aos processos de busca da eficiência energética sejam conhecidas pelos técnicos que estão ou estarão direta ou indiretamente ligados a este setor.

Um dos métodos de combate aos desperdícios da energia elétrica empregados na indústria refere-se ao diagnóstico energético ou auditoria energética. Cabe ressaltar que conforme Nogueira (2001) o diagnóstico energético refere-se a um estudo superficial enquanto auditoria seria um estudo mais detalhado dos processos energéticos de uma planta industrial.

La Rovere (1985) complementa que a conservação de energia ainda é vista como um conjunto de medidas de alcance limitado, visando à eliminação de desperdícios flagrantes no consumo de energia. Na verdade, este é apenas um primeiro nível de conservação de energia, segundo uma conceituação mais abrangente que envolve a diminuição da quantidade de energia primária necessária para propiciar o consumo de um mesmo nível de energia útil e a construção de um estilo de desenvolvimento que implique num perfil mais baixo da demanda de energia útil para um mesmo dado padrão de satisfação das necessidades sociais.

Porém, para se fazer um bom trabalho de conservação de energia levando em consideração a sustentabilidade da sociedade é necessário que todos em uma empresa ou instituição se conscientizem do que é conservação de energia num sentido mais amplo. Um bom programa de conservação de energia não está apenas na troca de equipamentos obsoletos por mais modernos, mas sim na mudança de hábito das pessoas, como por exemplo, um simples ato de apagar as luzes ao sair de um recinto.

Para Ribeiro (2004) a eficientização no uso da energia elétrica apresenta-se como uma proposta para criação de uma usina virtual. Sabe-se que existem muitos desperdícios de energia elétrica nas empresas, e por conta disso, há a necessidade de mais geração de energia elétrica e em conseqüência, todos os passivos ambientais que ocasionam. Portanto, a eficientização no uso da energia elétrica poupa recursos naturais, diminui as emissões e é um investimento com retorno financeiro garantido.

Reis (2003) e Bagattoli (2005) comentam que graus de desenvolvimento comparáveis aos alcançados até o presente são possíveis sem que seja necessário um aumento semelhante na utilização de energia como se verificou no processo de desenvolvimento anterior. Isto quer dizer que, com um uso eficiente de formas renováveis de energia, é possível prosseguir com o desenvolvimento sem exercer maiores pressões sobre o ecossistema. 
"Todos os processos usam energia e quase todos podem usar menos." (ANDERSON 2005, p. 23) $)^{2}$

Saidel et al. (2001 apud BAGATTOLI 2005) enfatiza que o grande potencial da conservação de energia elétrica está justamente no uso final, ou seja, no consumidor. Udaeta (2001 apud BAGATTOLI 2005) reforça esta posição ao comentar que provém dos usos finais de energia os maiores e mais graves problemas ambientais, porquanto ainda não foram mudados hábitos e culturas ligadas ao consumo, que são os determinantes das enormes quantidades de energia despendidas pela sociedade.

Deve-se ressaltar, porém, segundo La Rovere(1985) a necessidade de se efetuar uma análise da unidade industrial no que diz respeito à mesma permitir sua operação em faixas de eficiência adequadas, em seu processo produtivo, sua localização geográfica, sua gestão energética, seus planos de expansão e a qualificação de seus recursos humanos.

\subsection{MOTORES ELÉTRICOS E TRANSFORMADORES}

Nos ambientes industriais, de acordo com Bortoni \& Santos (2001), os motores elétricos e os transformadores representam de 50 a $70 \%$ das cargas industriais de modo que estes representam um grande potencial de conservação de energia.

Embora, de acordo com Bortoni \& Santos (2001), os motores de indução trifásicos, de uma forma geral, sejam máquinas intrinsecamente eficientes, estes se constituem de um grande potencial para a conservação de energia em função da grande quantidade de motores instalados e da aplicação ineficiente dos mesmos.

\subsection{ILUMINAÇÃO}

Em termos de sistemas de iluminação, estes representam $1 \%$ de toda energia elétrica consumida no setor industrial e 3,3\% no Brasil. Conforme Yamachita \& Haddad (2001), a iluminação excessiva é comum no Brasil. Uma combinação de lâmpadas, reatores e refletores eficientes, associados a hábitos saudáveis na sua utilização, podem ser aplicados para reduzir o consumo de energia elétrica. Como regra básica, a utilização de um sistema de iluminação eficiente consiste em utilizar apenas a luz necessária para cada atividade a ser desenvolvida.

Almeida (1995) e Padilla (2001) recomendam a exploração da iluminação natural sempre que possível de forma a reduzir o consumo de energia elétrica, porém, esta obtenção de eficiência é sempre de menor custo em novas construções. Em construções já existentes, o aproveitamento da iluminação natural pode gerar um alto custo inicial.

\footnotetext{
${ }^{2}$ Traduzido pelo autor: "All processes use energy and almost all could use less"
} 


\subsection{SISTEMAS DE REFRIGERAÇÃO E VENTILAÇÃO}

A refrigeração industrial, a exemplo do ar condicionado tem como objetivo a refrigeração de alguma substância ou meio. Os componentes básicos destes equipamentos não diferem entre si, há, contudo um predomínio do ar condicionado sobre a refrigeração industrial comparando-se o número de unidades instaladas e o volume de vendas.

Este predomínio em termos de número de equipamentos instalados faz com que o ar condicionado, de acordo com Pirani et al (2001), seja o maior responsável pelos picos de demanda de energia elétrica em instalações comerciais. No período vespertino, quando o ar condicionado torna-se necessário para manter temperaturas confortáveis, este aumento de demanda de energia soma-se àquela já causada pela iluminação, equipamentos, computadores e outros usuários.

\subsection{SISTEMAS DE BOMBEAMENTO}

As cargas representadas pelas bombas de fluxo e ventiladores das instalações industriais possuem de acordo com Viana (2001) um ponto de melhor funcionamento para uma determinada vazão, altura e rotação, em que suas perdas inerentes do melhor escoamento são mínimas. Porém, a maioria das instalações industriais exige da bomba ou do ventilador a operação fora deste ponto de melhor funcionamento, principalmente em função da necessidade de variação da vazão.

\subsection{CALDEIRAS E FORNOS INDUSTRIAIS}

A utilização eficiente da energia térmica em fornos ou caldeiras promove, além da redução de custos e do desperdício de energia, uma série de vantagens ambientais, principalmente em função de que, conforme Martins (2001), cerca de 53\% da energia total consumida pela indústria advém de combustíveis, sendo o restante suprido pela energia elétrica.

\subsection{CONTROLE DE DEMANDA}

O controle de demanda, realizado por um equipamento denominado "controlador de demanda" consiste em manter a demanda de energia elétrica do consumidor o mais próximo possível dos valores contratados, mas evitando qualquer tipo de ultrapassagem em relação aos mesmos. Com isto, além do consumidor pagar apenas pela demanda que é usada, contribui-se para a diminuição do desperdício. 
Segundo Bagattoli (2005), este controle mais direto do consumo da energia pode ser obtido, consistindo em fixá-lo em um determinado valor máximo e determinar quais as cargas, em que seqüência e por quanto tempo poderão ser desligadas manual ou automaticamente a fim de manter o teto estipulado de consumo.

\subsection{SISTEMAS PNEUMÁTICOS}

Beyene (2005) em uma pesquisa realizada em empresas dos EUA percebeu que a principal causa de desperdícios em sistemas pneumáticos encontra-se em vazamentos. Em muitos casos a geração de ar comprimido em pressão superior a necessária forçam o compressor a trabalhar além do necessário, haja vista que a pressão é rebaixada nos pontos de utilização. A diminuição da pressão de ar na geração contribui para a eficiência energética do sistema.

Ormer (2003) ressalta que a energia pneumática é mais cara do que se pensa, para cada 8 HP de energia elétrica converte-se em $1 \mathrm{HP}$ de trabalho com o ar comprimido de forma que os compressores de ar acionados por motores elétricos usam uma surpreendentemente quantia de energia durante os anos em que eles estão em operação. Não é incomum o custo anual de operação de um compressor se igualar ao seu preço de compra inicial.

\section{RESULTADOS OBTIDOS}

Para a execução deste trabalho optou-se basicamente pela inspeção visual nas instalações, visitas e análise de medições nas instalações efetuadas pelos profissionais da área de manutenção da empresa. Assim, puderam-se identificar os principais setores consumidores de energia elétrica de forma a permitir a sugestão de medidas de eficiência energética.

Tabela 1: Principais cargas instaladas na empresa

\begin{tabular}{l|c|c}
\hline \hline \multicolumn{1}{c|}{ Tipo de Carga } & Potência Instalada (kW) & Percentual (\%) \\
Motores elétricos & $1.447,37$ & 37,23 \\
Fornos & $1.975,00$ & 50,81 \\
Compressores de Ar & 228,16 & 5,87 \\
Condicionadores de Ar & 40,62 & 1,04 \\
Iluminação & 162,99 & 4,19 \\
Computadores & 33,25 & 0,86 \\
Total & $3.887,39$ & 100,0 \\
\hline \hline
\end{tabular}

Fonte: Empresa pesquisada: Metalúrgica Riosulense S.A. 


\subsection{SETORES ADMINISTRATIVOS}

As maiores alternativas para redução no consumo de energia elétrica neste setor devese ao aspecto comportamental e ao aspecto físico da instalação.

Na empresa os setores administrativos podem ser divididos em dois grupos: administração antiga e administração nova. A administração antiga apresenta setores dos escritórios junto ao prédio da usinagem enquanto que a administração nova apresenta setores dos escritórios em um prédio construído recentemente e que traz consigo conceitos de eficiência energética aplicada ao projeto.

A promoção da eficiência energética na administração nova estará diretamente relacionada aos aspectos comportamentais dos funcionários no que diz respeito ao uso dos aparelhos dos aparelhos de ar condicionado, computadores e iluminação. Na administração antiga, além dos aspectos comportamentais, a promoção da eficiência energética deverá estar alicerçada na substituição, manutenção ou redimensionamento de equipamentos ligados à iluminação, microcomputadores e aparelhos de ar condicionado.

\subsection{MICROCOMPUTADORES}

Os microcomputadores desempenham um papel fundamental nas indústrias hoje e já possuem instalados em seus sistemas um gerenciador de energia que, de acordo com Santos et al (2005) não chega a ser ativado pelos usuários. Este gerenciamento pode produzir uma sobrevida de $39 \%$ aos equipamentos, bem como, uma redução no consumo de energia que pode chegar a aproximadamente $28 \%$ apenas considerando o montante de energia utilizado na alimentação do PC.

Dentre os microcomputadores verificados na empresa pôde-se constatar esta função desativada na grande maioria dos microcomputadores.

Considerando-se um consumo equivalente à carga instalada e que todos os microcomputadores estejam com o modo de economia desativado, ou seja, um consumo mensal aproximado de $14,25 \mathrm{kWh}$, uma redução de $28 \%$ no consumo equivaleria a $4 \mathrm{kWh}$ aproximadamente.

\subsection{SISTEMAS DE AR CONDICIONADO}

Os sistemas de ar condicionado não interferem no processo de produção, mas sim, interferem no conforto dos funcionários envolvidos nas áreas administrativas. De uma forma geral as unidades condicionadoras de ar são máquinas adquiridas recentemente onde a questão comportamental no uso do sistema de ar condicionado será o fator predominante na obtenção da eficiência energética em relação a estes equipamentos. 


\subsection{ILUMINAÇÃO INDUSTRIAL}

A iluminação do parque fabril constitui aproximadamente $4,2 \%$ da carga instalada na empresa equivalente a $163 \mathrm{~kW}$, e desta forma considera-se responsável por $4,2 \%$ do consumo mensal de energia elétrica equivalente a $66.500 \mathrm{kWh}$.

Tomando-se como referência a menor possibilidade de eficientização efetuando-se a troca da iluminação fluorescente da administração, expedição, usinagem, fundição e iluminação externa com base nos valores de consumo dos catálogos dos fabricantes de equipamentos luminotécnicos, chega-se a uma diminuição no consumo mensal de aproximadamente $30 \%$, ou seja, $19.950 \mathrm{kWh}$.

Com a instalação adequada das telhas translúcidas nos setores produtivos estima-se uma diminuição no consumo mensal com o desligamento de lâmpadas equivalente a $1,5 \%$, ou seja, $1.000 \mathrm{kWh}$.

O resultado final será uma redução equivalente a $31,5 \%$ do consumo mensal de energia elétrica com sistemas de iluminação equivalente a 20.950kWh.

\subsection{MOTORES ELÉTRICOS}

Respondendo por aproximadamente $37,3 \%$ da carga instalada da empresa, os motores elétricos estão distribuídos pelo parque fabril nos diversos setores da empresa. Quase a totalidade dos motores instalados apresenta a característica de motor Standard, ou seja, não são motores de alto rendimento. Cerca de $90 \%$ dos motores apresentam potência igual ou inferior a $10 \mathrm{CV}$ e $70 \%$ dos motores apresentam idade igual ou inferior a 10 anos. Em medições efetuadas na empresa foi constatado problemas de dimensionamento em inúmeros motores que chegavam a limites inferiores de carregamento de $30 \%$.

Conforme mencionado, os motores elétricos representam aproximadamente $37,3 \% \mathrm{da}$ carga instalada na empresa, e considera-se, que equivalem a 37,3\% do consumo mensal em torno de $590.500 \mathrm{kWh}$. O uso ou substituição dos motores Standard por motores de alto rendimento pode levar a uma economia mensal de $25.400 \mathrm{kWh}$.

A economia de energia através de melhorias sistêmicas como redimensionamento de motores ou melhorias no processo pode levar a uma economia mensal de $23.325 \mathrm{kWh}$. A economia global, ou seja, a soma da economia que pode ser gerada pela substituição dos motores com a economia que pode ser gerada com melhorias sistêmicas chega a $48.725 \mathrm{kWh}$ que equivale a aproximadamente $8,3 \%$ do atual consumo mensal de energia considerado. 


\subsection{TRANSFORMADORES}

A principal metodologia a ser empregada pela empresa na eficientização dos processos consiste num estudo mais aprofundado sobre as cargas pertinentes a cada transformador de forma a obter uma distribuição de cargas que permita alternar o uso de transformadores que estejam ligados em paralelo e com baixo carregamento.

Com um fator de perda a vazio equivalente a $1 \%$ de sua potência que equivale a uma participação de $0,12 \%$ no consumo total da empresa, obtém-se uma redução de aproximadamente $1.900 \mathrm{kWh}$ no consumo de energia elétrica sem considerar os benefícios para a rede de distribuição e a qualidade de energia da empresa.

\subsection{CABOS DE ALIMENTAÇÃO E DISTRIBUILCÃO}

Está sendo adotada pela empresa uma política de adequação dos painéis elétricos à norma NR-10 - Segurança em Instalações e Serviços de Eletricidade, de forma que todos os painéis da empresa estão sendo auditados e seus circuitos (fiação e proteção) estão sendo recalculados e medidos de forma a verificar além do carregamento e dimensionamento, possibilidades de melhor alocação de circuitos pelo parque fabril.

Dos circuitos analisados na empresa, não foram encontrados problemas relacionados diretamente com dimensionamento ou instalação incorretos.

\subsection{FORNOS DE INDUÇÃO E CORREÇÃO DO FATOR DE POTÊNCIA}

Com relação a correção do fator de potência, a empresa possui em seu parque fabril células capacitivas distribuídas pontualmente nas maiores cargas (fornos e motores), bem como, bancos automáticos para correção do fator de potência.

Com relação aos fornos de indução, a sua eficientização se resume principalmente a obtenção de eficiência no processo e não ao equipamento propriamente dito.

O aumento da eficiência energética com o intuito de diminuir o consumo específico dos fornos pode promover uma redução no consumo através do melhor aproveitamento do processo produtivo. Sem alterar as características do equipamento obtém-se um ganho de até $81.237 \mathrm{kWh}$ por mês em função de pequenas medidas de eficientização do processo. 


\subsection{REDES DE AR COMPRIMIDO}

A localização dos compressores hoje não favorece a captação do ar comprimido de forma que, $\mathrm{o}$ ar aspirado pelo compressor apresenta temperatura elevada que vem a acarretar um consumo maior de energia por parte do compressor e da unidade de tratamento. Além disso, os vazamentos também têm grande parcela nas perdas decorrentes da geração do ar comprimido na empresa.

Cabe aqui a análise dos equipamentos alimentados por ar comprimido, a verificação da real necessidade da pressão de trabalho e um estudo para a melhoria das instalações dos compressores. Espera-se com a adoção das medidas referentes a melhoria das instalações dos compressores sobre os aspectos de aspiração e tratamento do ar comprimido um ganho de aproximadamente $2.600 \mathrm{kWh}$.

\section{CONSIDERAÇÕES FINAIS}

Com o enfoque no conceito de diagnóstico energético, este trabalho pode levantar dados com o intuito de orientar os responsáveis pelo gerenciamento de energia das empresas do setor metal-mecânico a obter eficiência energética em suas plantas.

As possibilidades de economia de energia alcançam estimativamente o valor de 155.416kWh equivalente a 9,8\% do consumo da empresa pesquisada. Observa-se que apenas o equivalente à metade deste valor faz referência a valores considerados concretos e mensuráveis de serem realizados por dependência da situação ou instalação dos equipamentos sendo a outra metade, principalmente relacionados ao consumo dos fornos, decorrentes de modificações ou melhorias no processo produtivo.

Desta forma podemos definir como principal fator contribuinte para o desperdício de energia, ou melhor, para a baixa eficiência da planta industrial seus procedimentos de produção, havendo a necessidade de revê-los dentro das possibilidades e limitações dos equipamentos.

A empresa pesquisada poderá promover uma economia de energia de aproximadamente $9,8 \%$ em relação ao seu consumo. Se considerarmos o consumo proporcional a carga instalada e extrapolarmos este valor para a demanda da empresa, obtemos um valor equivalente a 353kVA.

A extrapolação destes valores para as demais empresas consumidoras do Alto Vale não levará em consideração as possibilidades de economia de energia nos fornos, haja vista que, conforme observado a economia dos fornos faz referência direta aos aspectos operacionais e produtivos do processo em que eles estão envolvidos tornando assim possível uma economia considerada de $4,7 \%$ de energia nestas empresas. 
Assim, extrapolando este valor aos cinco maiores consumidores de energia elétrica setor metal-mecânico do Alto Vale do Itajaí, podemos alcançar valores de 649kVA de economia de energia por mês.

Esta energia economizada atua no sistema elétrico como uma usina virtual de forma a atuar como inibidor da necessidade de novos investimentos em geração e distribuição de energia.

Finalmente, demonstra-se que a eficientização é possível sem a necessidade de alteração na demanda de produção, pelo contrário, pode-se até obter uma melhor eficiência da planta podendo-se obter como conseqüência o aumento de produção, a diminuição de gastos com energia e, através da divulgação de resultados, o maior comprometimento dos funcionários com a empresa.

\section{REFERÊNCIAS}

ALMEIDA, M. A. Análise de cenários de conservação de energia elétrica para o Brasil. 1995. Dissertação (Mestrado em Ciências em Planejamento Energético) - Universidade Federal do Rio de Janeiro, Rio de Janeiro, 1995.

ANDERSON, C. Easier energy efficiency. Process Engineering. [s.I.], v. 86, n. 4, p. 23 25, 2005.

BAGATTOLI, S. G. Gestão estratégica da energia elétrica e seus reflexos no desenvolvimento regional. 2005. Dissertação (Mestrado em desenvolvimento regional) - Universidade Regional de Blumenau, Blumenau, 2005.

BEYENE, A. Energy efficiency and industrial classification. Energy Engineering. [s.l.], v. 102, n. 2, p. $59-80,2005$.

BORTONI, E. C.; SANTOS, A. H. M. Acionamentos com motores de indução trifásicos. In: HADDAD, J.; MARTINS, A. R. S.; MARQUES, M. (Coord.). Conservação de energia: Eficiência energética de instalações e equipamentos. 2. ed. Itajubá: EFEl, 2001.

FERREIRA. F. P. Perfil do produto energia elétrica da empresa CELESC - Agência Regional de Rio do Sul. 2003. Monografia (Bacharelado em Ciências Econômicas) Faculdade de Ciências Econômicas, Universidade Regional de Blumenau, Blumenau, 2003. 
LA ROVERE, E. L. Conservação de energia em sua concepção mais ampla: Estilos de desenvolvimento a baixo perfil de consumo de energia. In: LA ROVERE, E. L.; ROSA, L. P.; RODRIGUES, A. P. Economia e Tecnologia da Energia. Rio de janeiro: Marco Zero, 1985.

MARTINS, A. R. S. Caldeiras e fornos. In: HADDAD, J.; MARTINS, A. R. S.; MARQUES, M. (Coord.). Conservação de energia: eficiência energética de instalações e equipamentos. 2. ed. Itajubá: EFEI, 2001.

NOGUEIRA, L. A. H. Auditoria energética. In: HADDAD, J.; MARTINS, A. R. S.; MARQUES, M. (Coord.). Conservação de energia: eficiência energética de instalações e equipamentos. 2. ed. Itajubá: EFEI, 2001.

ORMER, H. V. Oportunities abound for saving energy. Hydraulics \& Pneumatics. [s.I.], v. 56, n. 2, p. $32-36,2003$.

PADILLA, J. V. Diagnóstico energético e redução de custos com energia nas empresas. Eletricidade Moderna. São Paulo, v. 29, n. 333, p. 110 - 117, Dez. 2001.

PIRANI, M. J. et al. Refrigeração e ar condicionado. In: HADDAD, J.; MARTINS, A. R. S.; MARQUES, M. (Coord.). Conservação de energia: eficiência energética de instalações e equipamentos. 2. ed. Itajubá: EFEI, 2001.

RAUEN, F. J. Roteiros de investigação científica. Tubarão: UNISUL, 2002.

REIS, L. B. dos, Geração de energia elétrica: tecnologia, inserção ambiental, planejamento, operação e análise de viabilidade. 3. ed. Barueri: Manole, 2003.

SANTOS, C. M.; SAIDEL, A.; HENRIQUES; M. S. Economia de energia em escritórios pelo gerenciamento de microcomputadores. Eletricidade Moderna. São Paulo, v. 33, n. 373, p. $266-275$, Abr. 2005.

VIANA, A. N. C. Bombas de fluxo e ventiladores. In: HADDAD, J.; MARTINS, A. R. S.; MARQUES, M. (Coord.). Conservação de energia: Eficiência energética de instalações e equipamentos. 2. ed. Itajubá: EFEI, 2001.

YAMACHITA, R. A.; HADDAD, J. Iluminação. In: HADDAD, J.; MARTINS, A. R. S.; MARQUES, M. (Coord.). Conservação de energia: eficiência energética de instalações e equipamentos. 2. ed. Itajubá: EFEI, 2001. 


\section{SOBRE OS AUTORES}

Graduado em Engenharia Elétrica pela FURB em 2000. Pós graduação em
nível de especialização em Automação Industrial pela FURB em 2003. Pós
graduação em nível de mestrado em Engenharia Ambiental pela FURB em
2007. Engenheiro eletricista e sócio proprietário da Rioar Automação Ltda
de Rio do Sul- SC. Docente da Faculdade de Tecnologia SENAI Rio do Sul nas
disciplinas de Pneumática e hidráulica, máquinas elétricas e Manutenção
elétrica. Discente do curso de pós graduação em Gerenciamento de projetos
da Faculdade SENAC de Rio do Sul.
E-mail: sidnei@rioar.ind.br
Raduenz

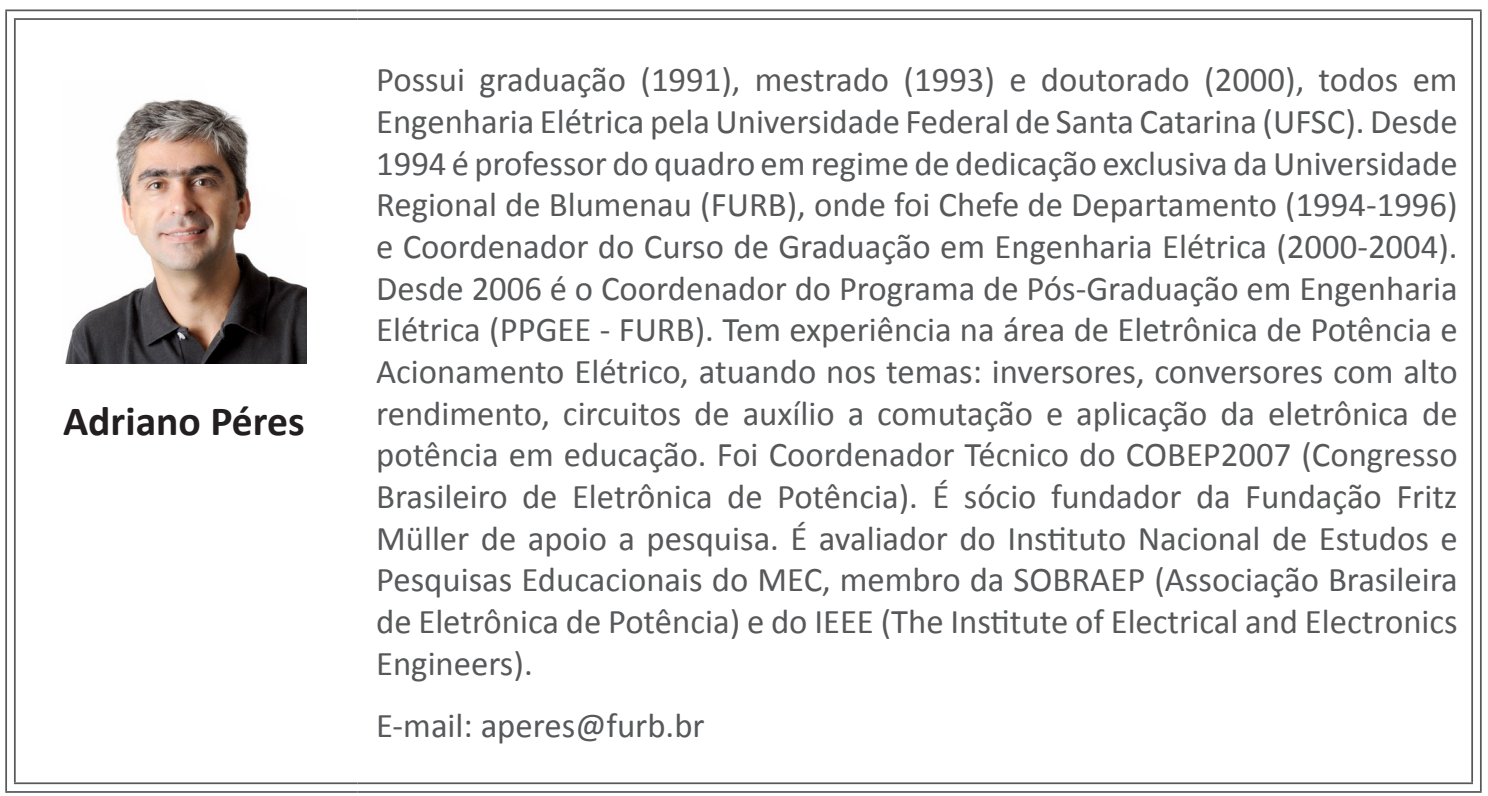

Possui graduação em Engenharia Elétrica (1987), especialização em
Empreendedorismo na Engenharia (2000), mestrado em Engenharia Elétrica
(1990) e doutorado em Engenharia Elétrica (1999), todos pela Universidade
Federal de Santa Catarina. É professor titular da Universidade Regional de
Blumenau, Conselheiro da Fundação Fritz Müller e Docente Avaliador
do Instituto Nacional de Estudos e Pesquisas Educacionais. É membro da
SOBRAEP (Associação Brasileira de Eletrônica de Potência) e do IEEE (The
Institute of Electrical and Electronics Engineers). Tem experiência na área de
Engenharia Elétrica, com ênfase em Eletrônica Industrial, Sistemas e Controles
Eletrônicos. Atuando principalmente nos seguintes temas: Conversores
Multiníveis, Conversores CC-CC, Comutação Suave, Fontes de Alimentação.
E-mail: edudes@furb.br

